\title{
Utility of OCT3/4, TSPY and $\beta$-catenin as biological markers for gonadoblastoma formation and malignant germ cell tumor development in dysgenetic gonads
}

\author{
Icela Palma ${ }^{\mathrm{a}, \mathrm{b}}$, Nayely Garibay ${ }^{\mathrm{c}}$, Rocio Pena-Yolanda ${ }^{\mathrm{d}}$, Alejandra Contreras ${ }^{\mathrm{e}}$, Atlantida Raya $^{\mathrm{f}}$, \\ Carolina Dominguez ${ }^{\mathrm{g}}$, Mirna Romero ${ }^{\mathrm{a}}$, Gerardo Aristi ${ }^{\mathrm{h}, \mathrm{i}}$ and Gloria Queipo ${ }^{\mathrm{c}, \mathrm{h}, *}$ \\ ${ }^{a}$ Molecular and Cellular Morphology Laboratory, Escuela Superior de Medicina, Instituto Politécnico Nacional, \\ Mexico City, Mexico \\ ${ }^{\mathrm{b}}$ Morphology Department, Facultad de Medicina Veterinaria y Zootecnia, UNAM, Mexico City, Mexico \\ ${ }^{\mathrm{c}}$ Human Genetics Department, Hospital General de México, Mexico City, Mexico \\ d Pathology Department, Hospital Infantil de México-Federico Gómez, Mexico City, Mexico \\ e Biology Development Department, Hospital Infantil de México-Federico Gómez, Mexico City, Mexico \\ ${ }^{\mathrm{f}}$ Urology Department, Hospital Infantil de México-Federico Gómez, Mexico City, Mexico \\ ${ }^{g}$ Endocrinology Department, Hospital Infantil de México-Federico Gómez, Mexico City, Mexico \\ ${ }^{\mathrm{h}}$ Facultad de Medicina Universidad Nacional Autónoma de México, Mexico City, Mexico \\ i Pathology Department, Hospital General de México, Mexico City, Mexico
}

\begin{abstract}
.
BACKGROUND: Gonadoblastoma (GB) is regarded as an in situ form of germ cell tumor in dysgenetic gonads, and $30 \%$ of patients with GB develop a dysgerminoma/seminoma tumor.

OBJECTIVE: Determine whether OCT3/4 and $\beta$-catenin are expressed in dysgenetic gonads before GB development and whether TSPY participates in the OCT3/4- $\beta$-catenin pathways in the malignant invasive behavior.

METHODS: dysgenetic gonads of Disorders of sex differentiation (DSD) patients with mixed gonadal dysgenesis were analyzed by immunohistochemistry and immunofluorescence for comparison with GB and dysgerminoma/seminoma.

RESULTS: Our results suggest that the development of GB is secondary to the interaction of OCT3/4 and TSPY, that $\beta$-catenin does not participate in this process.

CONCLUSIONS: The use of this biological markers detects the potential high risk gonads.
\end{abstract}

Keywords: Gonadoblastoma, OCT3/4, TSPY, $\beta$-catenin, dysgenetic gonads, mixed gonadal dysgenesis

\section{Background}

Gonadoblastoma (GB) is regarded as an in situ form of germ cell tumor in dysgenetic gonads (type

* Corresponding author: Gloria Queipo, Human Genetics Department, Hospital General de Mexico, Mexico City, Mexico; Facultad de Medicina Universidad Nacional Autonoma de Mexico, Mexico City, Mexico. Dr. Balmis 142 Col, Doctores CP 06766 Mexico DF. Tel.: +52 5527892000 (1278/1279); E-mail: gqueipo@nanolab.com.mx,gqueipo99@yahoo.com.
II GCTs). This type of tumor is thought to be a precursor to seminoma/dysgerminoma tumors. It almost exclusively affects a subset of patients with disorders of sex differentiation (DSD) [5,7]. In $35 \%$ of GB cases, overgrowth of the germinal component leads to dysgerminoma/seminoma [8]. The TSPY gene (testisspecific protein, Y encoded) localized within the $G B Y$ locus (gonadoblastoma locus on the $\mathrm{Y}$ chromosome) has been shown to be involved in the multistep transformation of germ cells to GB [3,13]. However, the 
Table 1

Tissue samples histopathology, and biological markers localization

\begin{tabular}{|c|c|c|c|c|c|c|}
\hline Case & Gonadal histopatolgy & ОСТ3/4 & TSPY & B-catenin & Co-localization TSPY/OCT3/4 & Co-localization TSPY/B-catenin \\
\hline 1 & UGT/SG & $(+)$ & $(+)$ & $(-)$ & $(+)$ & $(-)$ \\
\hline 2 & DT & $(+)$ & $(+)$ & $(-)$ & $(-)$ & $(-)$ \\
\hline \multirow[t]{2}{*}{3} & (R) SG & $(-)$ & $(-)$ & $(-)$ & $(-)$ & $(-)$ \\
\hline & (L) SG & $(-)$ & $(-)$ & $(-)$ & $(-)$ & $(-)$ \\
\hline \multirow[t]{2}{*}{4} & (R) ${ }^{*} \mathrm{UGT} / \mathrm{SG}$ & $(+)$ & $(+)$ & $(+)$ & \multirow{2}{*}{$(+)$} & $(+)$ \\
\hline & (L) DT & $(+)$ & $(+)$ & $(-)$ & & $(-)$ \\
\hline 5 & DT & $(+)$ & $(+)$ & $(+)$ & $(+)$ & $(+)$ \\
\hline 6 & (L)UGT/SG & $(+)$ & $(+)$ & $(-)$ & $(-)$ & $(-)$ \\
\hline 7 & DT & $(+)$ & $(+)$ & $(-)$ & $(-)$ & $(-)$ \\
\hline 8 & DT & $(+)$ & $(+)$ & $(-)$ & $(-)$ & $(-)$ \\
\hline 9 & UGT/SG & $(+)$ & $(+)$ & $(-)$ & $(+)$ & $(-)$ \\
\hline 10 & DT & $(+)$ & $(+)$ & $(-)$ & $(-)$ & $(-)$ \\
\hline 11 & DT & $(-)$ & $(+)$ & $(-)$ & $(-)$ & $(-)$ \\
\hline 12 & UGT/SG & $(+)$ & $(-)$ & $(-)$ & $(+)$ & $(+)$ \\
\hline 13 & DT & $(+)$ & $(+)$ & $(-)$ & $(-)$ & $(-)$ \\
\hline 14 & $\begin{array}{l}\text { (R)GB } \\
\text { (L)GB }\end{array}$ & $(+)$ & $(+)$ & $(+)$ & \multirow[t]{2}{*}{$(+)$} & \multirow[t]{2}{*}{$(+)$} \\
\hline 15 & DG & $(+)$ & $(+)$ & $(+/-)$ & & \\
\hline
\end{tabular}

${ }^{*} \mathrm{UGT}=$ tissue with burnt-out gonadoblastoma, DT $=$ dysgenetic testis, $\mathrm{SG}=$ streak gonad, UGT $=$ undifferentiated gonadal tissue, $\mathrm{R}=$ right, $\mathrm{L}=$ left, $\mathrm{GB}=$ gonadoblastoma, $\mathrm{DG}=$ dysgerminoma.

precise role that TSPY plays in GB development and its involvement in the malignant transformation are not clear [15]. OCT3/4 has been implicated in the GB oncogenic process, but the molecular details of OCT3/4 deregulation are still unknown $[6,12]$. Analysis of OCT3/4, E-cadherin and $\beta$-catenin showed that the proliferation of immature germ cells in GB may be due to the interaction between OCT3/4 and accumulated $\beta$-catenin in the nuclei of the immature germ cells, leading to the development of invasive behavior and the progression of GB into dysgerminoma/seminoma in dysgenetic gonads [4]. In the present study, to determine whether TSPY participates in the OCT3/4- $\beta$-catenin pathway in the dysgenetic gonad and whether OCT3/4 and $\beta$-catenin are expressed in the dysgenetic gonad, we analyzed 18 dysgenetic gonads from DSD patients with mixed gonadal dysgenesis and compared them with GB and dysgerminoma/seminoma tumors.

\section{Materials and methods}

Eighteen paraffin-embedded tissue samples from 15 pediatric patients with mixed gonadal dysgenesis or ambiguous genitalia and a 45, X/46, XY karyotype were studied. Tissue samples from two bilateral GB and one dysgenetic gonad with dysgerminoma/seminoma transformation were included. The use of the tissues was approved by the Institutional Bioethics Board. The analyses were performed using the classification of the World Health Organization. Formalin-fixed, paraffin-embedded sections were analyzed using immunohistochemistry and immunofluorescence (Table 1). The assays were performed in triplicate. Positive controls for $\beta$-catenin, TSPY and OCT3/4 were included in each experiment. The analyses were performed by an experienced pathologist, using the classification of the World Health Organization. The histological results were assessed by two scientists experienced in germ cell pathology (YRP and IP). Antigen-antibody complexes were detected using the avidin-biotin peroxidase method (KO679 LSAB+Sys/HRP kit, DakoCytomation, Carpinteria, CA) or with a secondary antibody conjugated to fluorescein isothiocyanate. The histological characteristics of the tissues revealed three of the four morphological patterns described by Martine Cools et al. [8]. Eight of the 18 samples were from dysgenetic testis (DT); germ cells in all 8 of the DT samples were confirmed by positive TSPY-staining. The second pattern, found in 5/18 samples, was streak tissue within undifferentiated gonadal tissue (UGT). UGT is characterized by germ cells that are not enclosed in seminiferous tubules or follicles organized in cord-like structures or by those without apparent organization. One of the UGTs contained a burnt-out gonadoblastoma. The third pattern observed in 2/18 cases was streak tissue. We also included one bilateral GB and one dysgerminoma as controls (Figs 1(A), (D), (G), (J)). In the rest of the samples, no GB or developing tumor was observed (Table 1). 


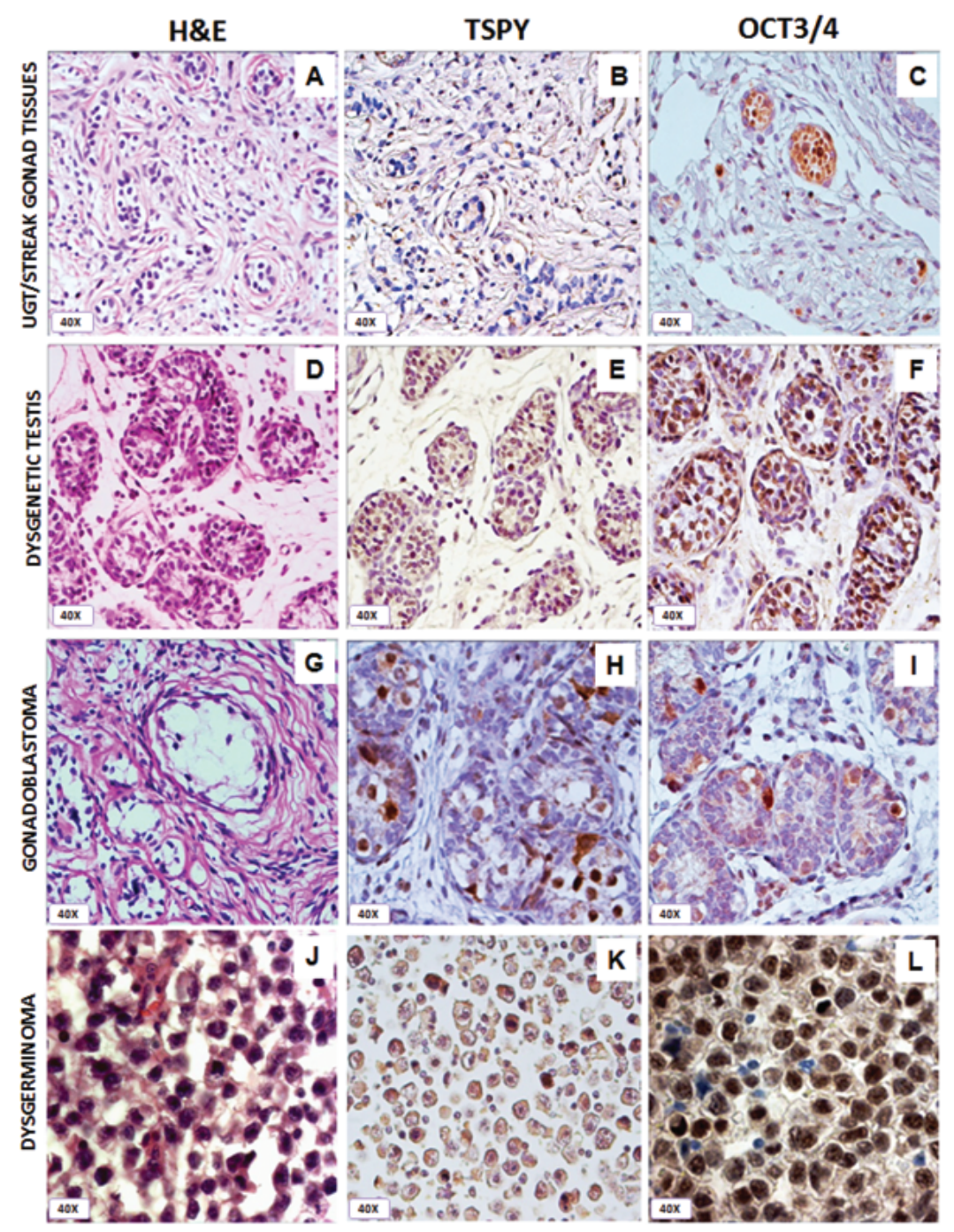

Fig. 1. H\&E light microscopy of a (A) streak gonad with an undifferentiated gonadal tissue (UGT) organized in cord-like structures, (D) dysgenetic testis containing seminiferous tubules consistent with a testicular differentiation pattern, $(\mathrm{G})$ a typical gonadoblastoma nest showing a mixture of mature and immature germ cells and (J) a dysgerminoma tumor. TSPY immunohistochemistry showing positive immunoreactive signals in the immature germ cells in an UGT (B), DT (E) and (H) positive signal in immature germ cells within a gonadoblastoma nest. and dysgerminoma (K) OCT3/4 in the same expression pattern as TSPY in UGT, DT, GB and dysgerminoma (C, F, I, L).

\section{Results}

In $14 / 18$ of the gonadal samples, the germ cells stained positive for OCT3/4; OCT3/4 immunoreactivity was detected in the nuclei of immature germ cells and was observed exclusively in the DT, UGT, and control tumors as well as in GB and dysgerminoma tumors (Figs 1(C), (F), (I), (L)). OCT3/4 protein was not detected in mature germ cells or the streak tissue. TSPY immunostaining was positive in 14/18 gonads. TSPY protein staining was strongly positive in the nuclei of the germ cells in DT, UGT, GB and dysger- minoma tissues. Some protein was also detected as a faint stain in the germ cell cytoplasm (Figs 1(B), (E), $(\mathrm{H}),(\mathrm{K}))$. As in the case of GB, TSPY was detected in the UGT tissue containing burnt-out gonadoblastoma, suggesting that OCT3/4 and TSPY are key proteins in the development of GB. The samples that were negative for TSPY were mainly those with streak regions lacking germ cells. To determine whether TSPY and OCT3/4 were colocalized in the nuclei of immature germ cells, confocal microscopy was performed. It showed that both proteins were colocalized in the immature germ cell nuclei in one dysgenetic testis, in 

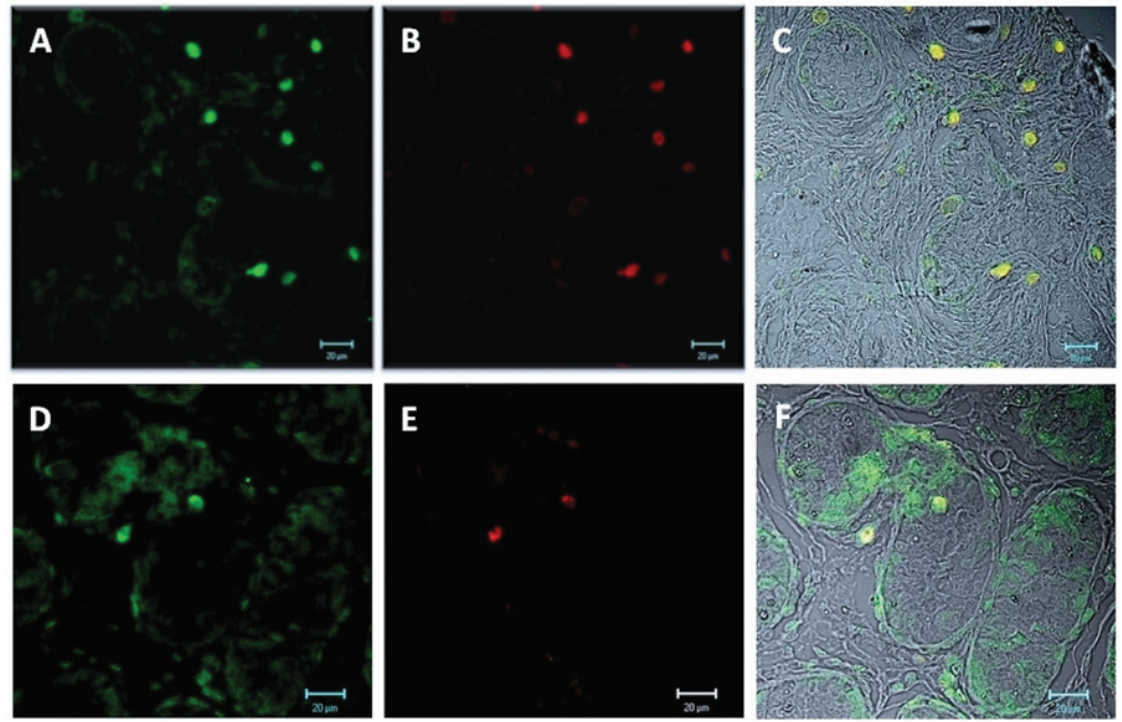

Fig. 2. Double-staining immunofluorescence and confocal analysis of dysgenetic testis positive for TSPY, OCT3/4 and $\beta$-catenin. (A) TSPY (green) localized in immature germ cells inside the seminiferous tubules, (B) OCT3/4 in the same tissues showing the same immunofluorescence pattern (red), (C) merged image and transmitted light micrograph optical transmission of the analyzed area indicating colocalization of both proteins in the nuclei of immature germ cells, (D) TSPY-positive immunofluorescence signal (green), (E) $\beta$-catenin (green) and (F) optical transmission and merged image showing colocalization of both proteins.

UGT and in GB (Figs 2(A)-(C)). Previous results have suggested that OCT3/4 and $\beta$-catenin participate in important steps during GB malignant transformation. Bcatenin immunoreactive regions were observed only in 3/18 dysgenetic gonads. Nuclear staining in immature germ cells was observed in one DT, in UGT/GB and in GB (Figs 3(A)-(J)). Interestingly, in our previous report, dysgerminoma showed diminished $\beta$-catenin expression. Gonads that were positive for $\beta$-catenin also expressed OCT3/4 and TSPY, which colocalized. All OCT3/4, TSPY positive gonads demonstrated expression of Ki67, a cell proliferative marker (Fig. 1P$\mathrm{S})$. Confocal microscopy showed colocalization of $\beta$ catenin and OCT3/4 in all the positively stained samples, as we reported previously. TSPY colocalized with $\beta$-catenin in $\beta$-catenin-positive cells (Fig. 2D-F). However, the majority of the dysgenetic gonads tested were negative for this marker; eliminating the possibility that $\beta$-catenin participates in gonadoblastoma formation.

\section{Discussion}

Pure GB is regarded as an in situ form of germ cell tumor that affects almost exclusively a subset of DSD patients with dysgenetic gonads. GB does not behave as a malignant lesion; nevertheless, ap- proximately $30 \%$ of all patients with gonadoblastoma develop a dysgerminoma/seminoma $[1,7,8]$. The age at diagnosis is variable, with approximately $94 \%$ of the cases being diagnosed during the second or third decades of life; we have demonstrated the presence of GB in infants [14]. It is important to identify a biological marker capable of detecting those dysgenetic gonads with a high potential for developing a tumor. Key proteins associated with germ cell tumor development, such as OCT3/4, $\beta$-catenin, TSPY and Ki67, were analyzed in 16 dysgenetic gonads and two germ cell tumors. GB originates from the surviving OCT3/4positive germ cells within undifferentiated gonadal tissue in the dysgenetic gonad [8]

We classified our dysgenetic tissue into three patterns (DT, UGT and streak gonad). It is important to emphasize that the streak tissue must be carefully examined to identify UGT in all the patients. In our samples, five streak tissues contained UGT (Figs 1(A)(C)). Gonadal biopsy identified as UGT or DT contained OCT3/4-positive cells, indicating a high risk for germ cell tumor formation because OCT3/4-positive cells are implicated in the GB oncogenic process. OCT3/4 is considered the most informative marker for the diagnosis of germ cell tumors $[9,11]$. In contrast, TSPY gene is the putative gene that predisposes dysgenetic gonads of intersex patients to develop gonadoblastomas. TSPY-positive immature germ cells 


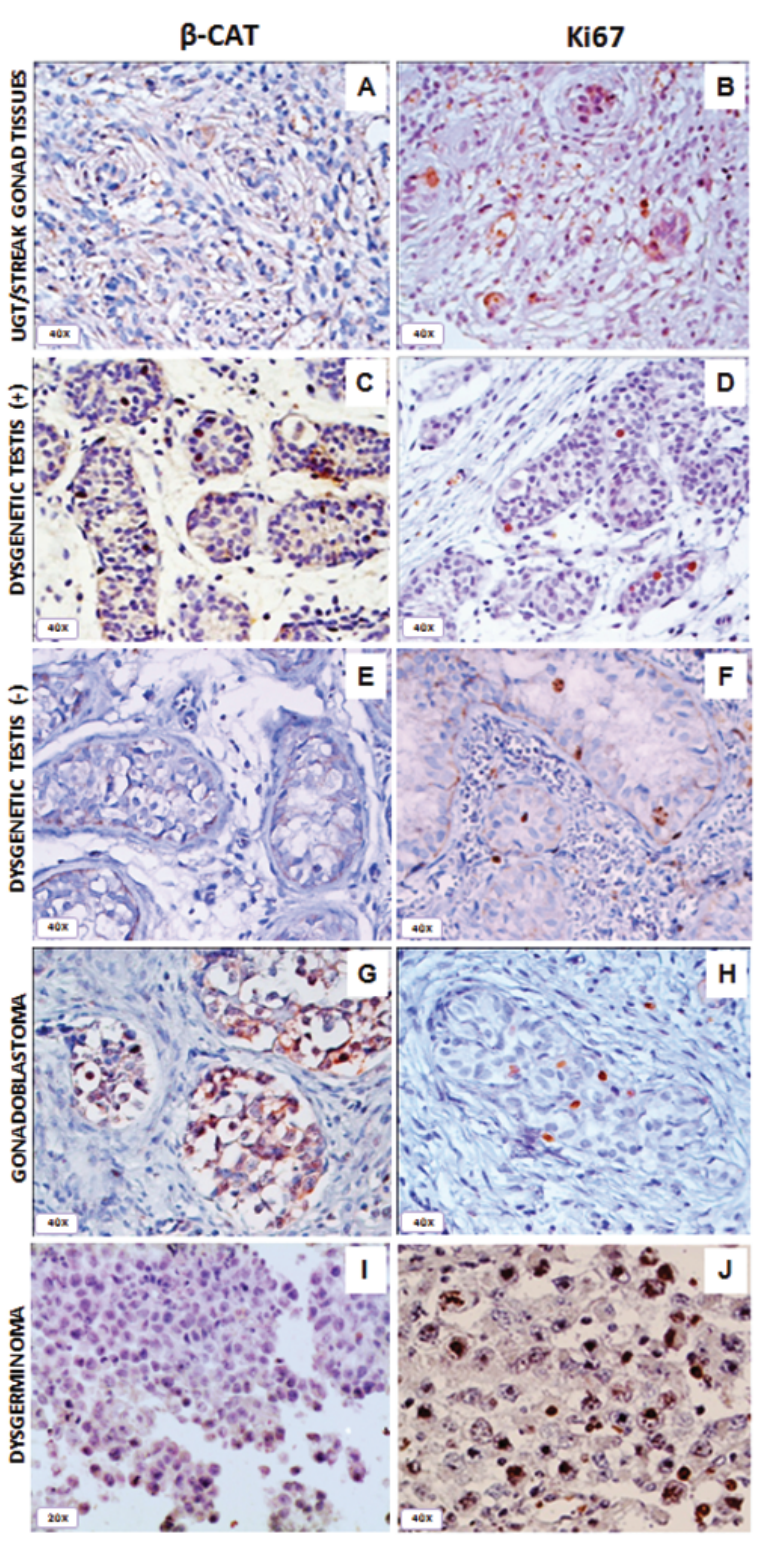

Fig. 3. $\beta$-catenin immunohistochemistry showing the three different patterns identified: (A) positive staining in immature germ cells in UGT tissue, (C) negative in most of DT, (E) positive pattern in DT $(\mathrm{G})$ strong immunoreactivity in gonadoblastoma, (I) positive but faint staining in dysgerminoma. Ki67 was used as a proliferative marker (B, D, F, H, and J).

were observed in only two dysgenetic gonads, one of which was in a UGT containing a burnt-out GB. Not all OCT3/4-positive cells showed the presence of TSPYpositive nuclei, suggesting that the interaction between OCT3/4 and TSPY is an important step in GB formation. The colocalization of both proteins in the nuclei of immature germ cells, together with the Ki67 proliferative marker, supports the idea that the interaction of these two proteins in the nuclei of immature germ cell leads to cellular proliferation and GB development (Fig. 2). This finding confirms that the study of these proteins are a significant diagnostic marker for GB, CIS/ITGCNU and seminomatous tumors [1, 9]. The abundant expression of TSPY in both gonadoblastomas and CIS/ITGCNU tissues further supports the concept of a common origin [13] (Figs 1(E)(H)). In the same way, the OCT3/4 transcription factor plays a pivotal role as a key regulator of pluripotency in the early stages of mammalian development [12]. Our observations suggest that in the dysgenetic gonad, OCT3/4 and TSPY nuclear overexpression are the key factors in the development of GB. The ectopic germ cells and the dysgenetic tissues require the presence of both proteins to proliferate. OCT3/4 expression in germ cell tumors and cancers of somatic origins suggests that it might have a proliferative function at the cellular level when it is ectopically expressed in these cells [13]. GB is not a common tumor; therefore, an insufficient number of cases have been analyzed. Previous research on $\beta$-catenin and OCT3/4 suggests that both proteins participate in the same oncogenic pathway during germ cell tumor development. The interaction between OCT3/4 and the $\beta$-catenin that accumulated in the nuclei of immature germ cells leads to the development of invasive behavior and the progression of GB into dysgerminoma/seminoma in dysgenetic gonads [14]. Here, the data show that $\beta$-catenin is expressed only in the nuclei of immature germ cells in the dysgenetic tissues that coexpressed OCT3/4 and TSPY. The remainder of the samples did not express $\beta$-catenin. This finding suggests that $\beta$-catenin participates only after the GB is established and is not involved in dysgenetic gonad progression to GB. In our previous study, we found that $\beta$-catenin expression is diminished in dysgerminoma tumors by comparison with colon adenocarcinoma; however, its colocalization with OCT3/4 suggests that both proteins participate in the same oncogenic pathway [4]. These observations distinguish $\beta$-catenin as a malignancy marker in the germ cells in which it is expressed and in dysgenetic tissues that are OCT3/4-TSPY-positive.

In conclusion, dysgenetic tissue expressing OCT3/4TSPY is associated with an extremely high risk for GB development, and both proteins are key players during GB development. The analysis of OCT4, SRY, TSPY and $\beta$-catenin expression in dysgenetic gonads may introduce modifications in the microenvironment that could contribute to a malignant transformation process. 
The presence of $\beta$-catenin suggests that this protein is linked to malignant transformation (Figs 2(D)-(F)) [2]. The presence of OCT3/4-TSPY in the gonadal biopsy tissues from DSD patients is an indicator of a high risk for GB, and $\beta$-catenin should be used as a marker for malignant germ cell tumors.

\section{Acknowledgments}

This work was performed in the Human Genetics Department at the Hospital General de México Eduardo Liceag, Facultad de Medicina UNAM. This work was supported by the Research Division of the Hospital General de México, CONACYT grant number 115440 .

\section{References}

[1] AM Kersemaekers, et al., Identification of germ cells at risk for neoplastic transformation in gonadoblastoma: an immunohistochemical study for OCT3/4 and TSPY, Hum Pathol 36 (2005), 512-21.

[2] B Bianco, KC Oliveira, AD Guedes, et al., OCT4 gonadal gene expression related to the presence of Y-chromosome sequences in Turner syndrome, Fertil Steril 94 (2010), 2347-9.

[3] F Schnieders, et al., Testis-specific protein, Y-encoded (TSPY) expression in testicular tissues, Hum Mol Genet 5 (1996), 1801-7.

[4] I Palma, et al., Participation of OCT3/4 and beta-catenin during dysgenetic gonadal malignant transformation, Cancer Lett 263 (2008), 204-11.
[5] JW Oosterhuis and LH Looijenga, Testicular germ-cell tumours in a broader perspective Nat Rev Cancer 5 (2005), 21022.

[6] L Cheng, et al., OCT4: Biological functions and clinical applications as a marker of germ cell neoplasia, J Pathol 211 (2007), 1-9.

[7] LH ooijenga, et al., Gonadal tumours and DSD, Best Pract Res Clin Endocrinol Metab 24 (2010), 291-310.

[8] M Cools, et al., Morphological and immunohistochemical differences between gonadal maturation delay and early germ cell neoplasia in patients with undervirilization syndromes, $\mathrm{J}$ Clin Endocrinol Metab 90 (2005), 5295-303.

[9] M Cools, et al., Gonadoblastoma arising in undifferentiated gonadal tissue within dysgenetic gonad, J Clin Endocrinol Metab 91 (2006), 2404-13.

[10] N Liu, et al., Genome-wide gene expression profiling reveals aberrant MAPK and Wnt signaling pathways associated with early parthenogenesis, J Mol Cell Biol, 2 (2010), 333-44.

[11] R Hersmus, et al., New insights into type II germ cell tumor pathogenesis based on studies of patients with various forms of disorders of sex development (DSD), Mol Cell Endocrinol 291 (2008), 1-10

[12] S Gidekel, et al., Oct-3/4 is a dose-dependent oncogenic fate determinant, Cancer Cell 4 (2003), 361-70.

[13] Y Li, ZL Tabatabai, et al., The Y-encoded TSPY protein: A significant marker potentially plays a role in the pathogenesis of testicular germ cell tumors, Hum Pathol 38 (2007), 147081.

[14] Y-R Peña, K Nieto, R Alvarez, I Palma, N Nájera, L Eraña, LM, S Kofman-Alfaro, G Queipo, Distribution of $Y$ chromosome-bearing cells in gonadoblastoma and dysgenetic testis in 45, X/46, XY infants, Mod Pathol 18 (2005), 439-45.

[15] YF Lau, Y Li and T Kido, Role of the Y-located putative gonadoblastoma gene in human spermatogenesis, Syst Biol Reprod Med 57 (2011), 27-34. 


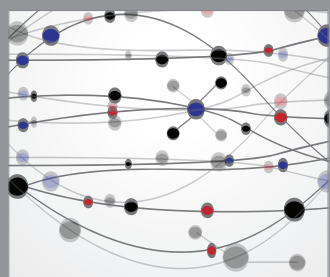

The Scientific World Journal
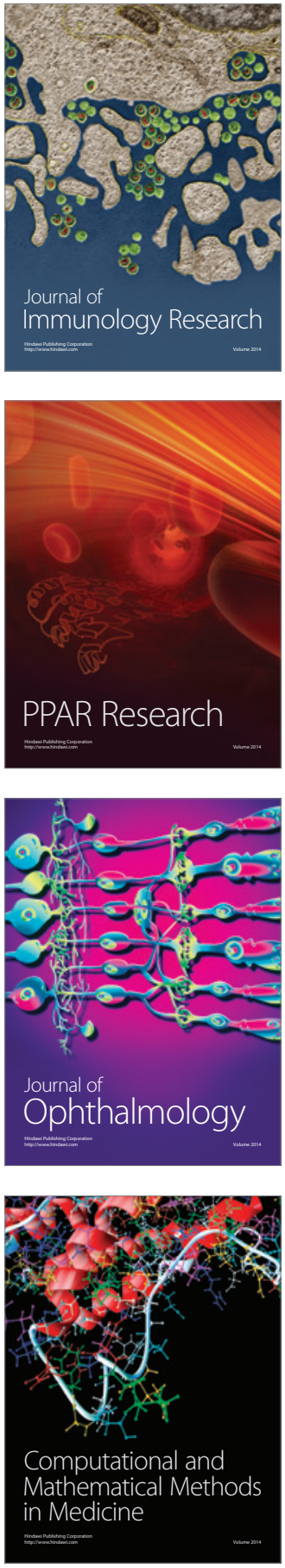

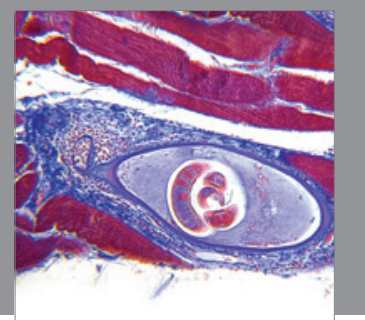

Gastroenterology

Research and Practice
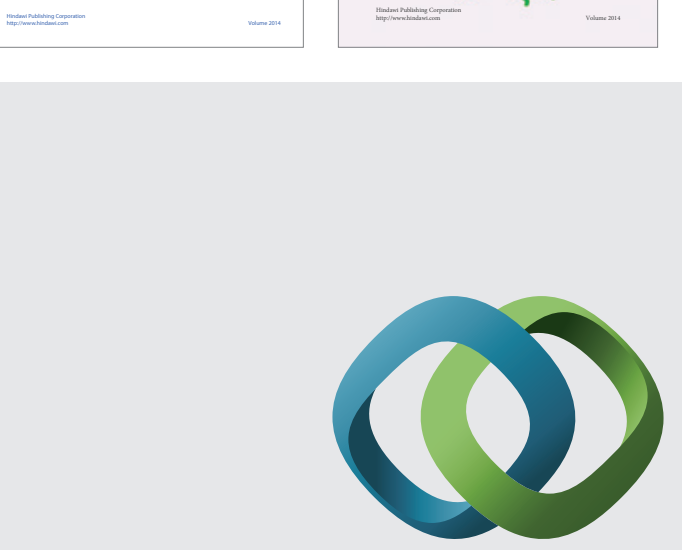

\section{Hindawi}

Submit your manuscripts at

http://www.hindawi.com
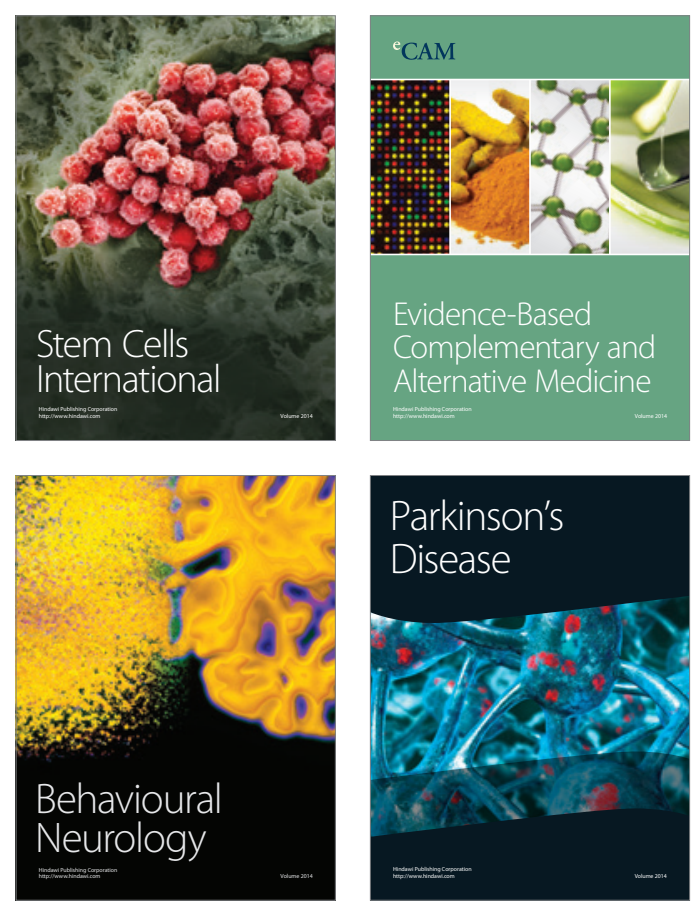

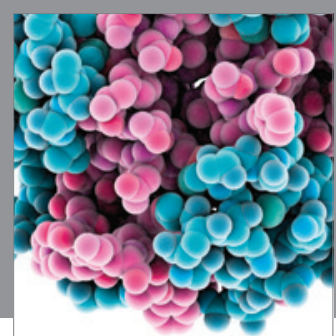

Journal of
Diabetes Research

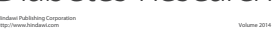

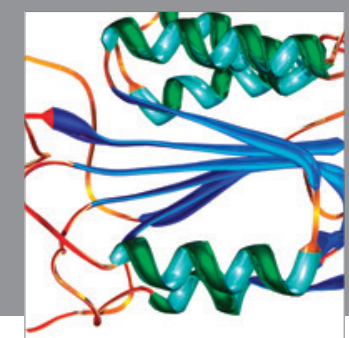

Disease Markers
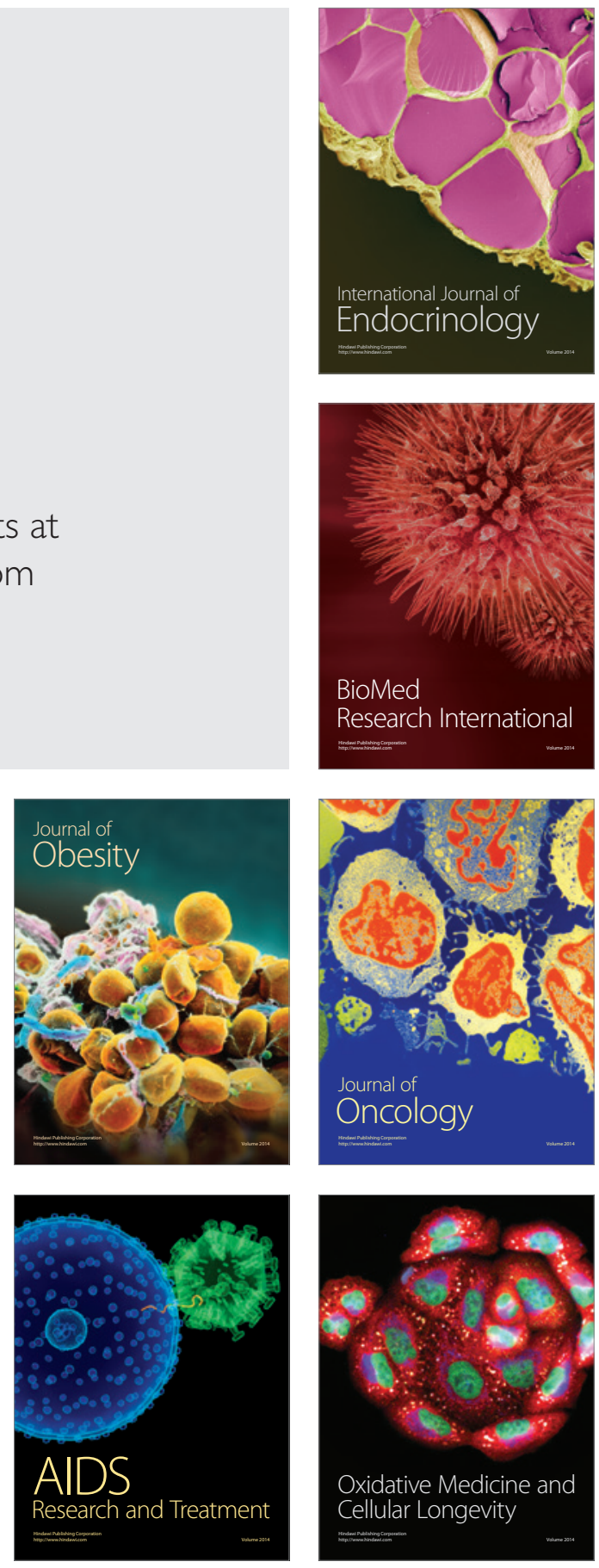